EPJ Web of Conferences 97,00025 (2015)

DOI: $10.1051 /$ epjconf/ 20159700025

(C) Owned by the authors, published by EDP Sciences, 2015

\title{
How much does the hadronic phase contribute to the observed anisotropic flow at the LHC?
}

\author{
Raimond Snellings ${ }^{1,2, a}$ \\ ${ }^{1}$ Nikhef National Institute for Subatomic Physics, Amsterdam, The Netherlands \\ ${ }^{2}$ Utrecht University, Princetonplein 5, 3584 CC Utrecht, The Netherlands.
}

\begin{abstract}
Elliptic flow signals the presence of multiple interactions between the constituents of the created matter in heavy-ion collisions. This includes possible contributions from the different phases, including the hadronic phase. In these proceedings I will first show that the energy dependence of elliptic flow, based on recent ALICE and STAR beam energy scan measurements, can largely be understood in terms of a boosted thermal system. In addition, a detailed comparison between the identified particle elliptic flow measured by the ALICE collaboration and viscous hydrodynamical model calculations with and without a hadronic afterburner is performed to constrain the possible effects of individual hadron-hadron re-interaction cross-sections.
\end{abstract}

\section{Introduction}

In the world around us, quarks and gluons do not exist as free particles because they are permanently bound into hadrons by the strong interaction. At very high temperatures and densities however, hadronic matter is expected to undergo a phase transition to a new state of matter, the QuarkGluon Plasma (QGP), where quark and gluon degrees of freedom are not anymore confined inside the hadrons. Collisions of heavy ions at RHIC and at the LHC energies produce a system with temperatures that are well above the strong phase transition temperature and are therefore a unique tool to create and study the complex properties of the QGP and the QCD phase transition to ordinary hadronic matter in the laboratory.

The experimental evidence that the QGP properties resemble those of a strongly interacting liquid has led to a consensus that much of the dynamical evolution of the created system can be modelled using viscous relativistic hydrodynamics. Figure 1 shows a cartoon of the time evolution of the collision and shows that after a short pre-equilibrium phase the system evolution is modelled in terms of viscous hydrodynamics.

It is observed that the bulk hadron yields in heavy-ion collisions are well described in thermal and statistical models assuming chemical equilibrium. These models successfully describe almost all bulk hadron yields, from center of mass energies of a few $\mathrm{GeV}$ to a few $\mathrm{TeV}$ [2]. In these models only two parameters, the chemical freeze-out temperature $T_{\text {chem }}$ and baryochemical potential $\mu_{B}$, are most relevant. The $T_{\text {chem }}$ extracted from thermal and statistical model fits to the data is close to the phase transition temperature obtained from recent lattice calculations and is found to saturate from RHIC up

\footnotetext{
a e-mail: R.J.M.Snellings@uu.nl
} 


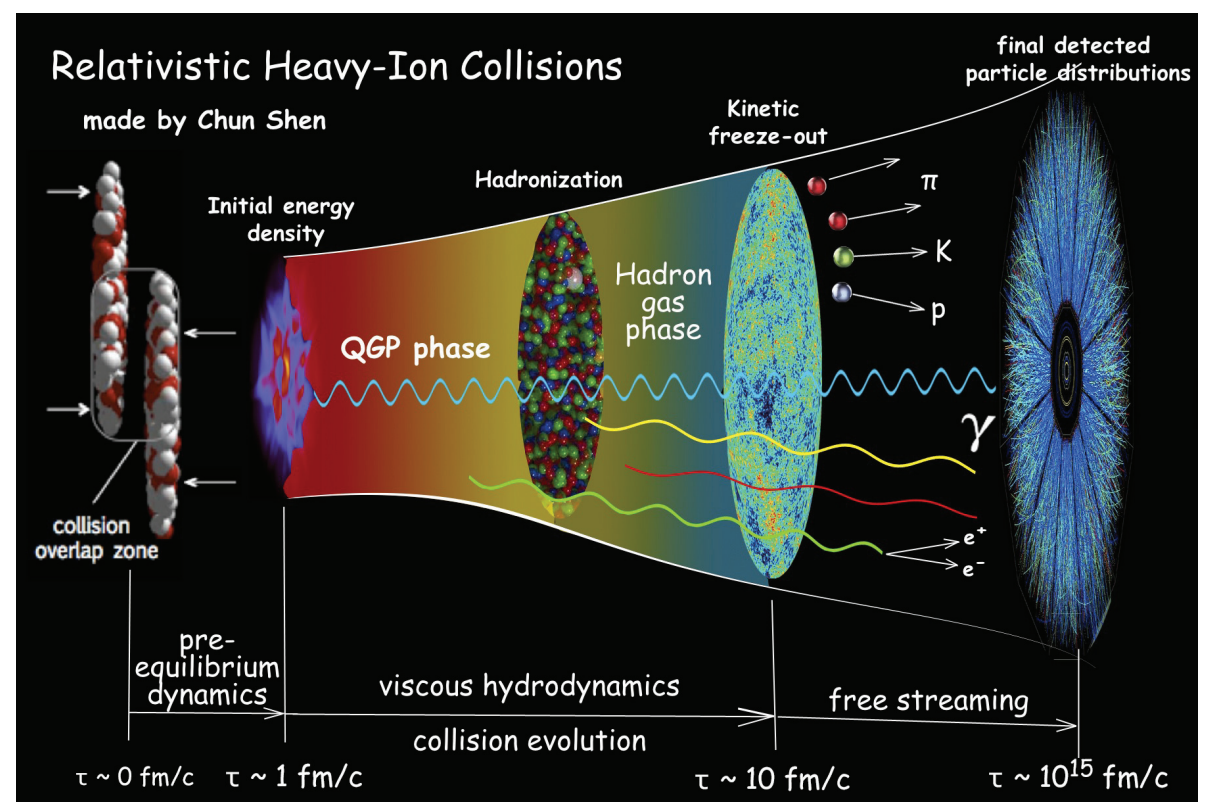

Figure 1. Cartoon of the time evolution of an ultra-relativistic heavy-ion collision. Figure taken from [1].

to LHC energies, where heavy-ions collide at an order of magnitude higher center of mass energy. In addition, measurements show that $T_{\text {chem }}$ is also constant as a function of collision centrality, which is naturally expected in these models.

Nevertheless, clear deviations from thermal and statistical model calculations are observed at the LHC in e.g. the changing $K^{* 0} / K^{-}$ratio as function of centrality [3] and in the proton and anti-proton yields [4] (although in this case the deviations are less than $3 \sigma$ ). For the success of the thermal and statistical model description this is a fly in the ointment and these deviations of the proton and anti-proton yields were part of a larger so-called proton puzzle at the LHC (at lower energies similar deviations are observed [5]).

A possible explanation for these deviations could be that statistical hadronization occurs out of chemical equilibrium [6], while another scenario could be modifications due to some inelastic hadronhadron final stage interactions (e.g. baryon-antibaryon annihilation [7]) at temperatures below $T_{\text {chem }}$.

In contrast to the bulk hadron yields, the momentum distributions of the finally emitted hadrons can not be described as a thermal system with $T_{\text {chem }}$ but instead as a boosted thermal system, with a kinetic freeze-out temperature $T_{\text {kin }}$ and radial flow velocity $\beta_{0}$. It is found that the observed $T_{\text {kin }}$ is smaller than $T_{\text {chem }}$, which is considered evidence for quasi-elastic resonance scattering in the hadronic phase.

If the created system stays close to kinetic equilibrium during the hadronic phase it can be modelled using viscous hydrodynamics, while accounting for chemical freeze-out at $T_{\text {chem }}$. An example of such a model is VISH2+1 [8, 9]. However, if the system becomes too dissipative in the hadronic phase viscous hydrodynamics breaks down, and in this case a microscopic hadron cascade model should provide a more reliable description. VISHNU [10] is an example of a model which matches a 
viscous hydrodynamical model VISH2 +1 to a hadron cascade model URQMD [11] for the dissipative hadronic phase.

In the following sections of these proceedings I will first show that the integrated and $p_{\mathrm{T}^{-}}$ differential elliptic flow for charged and identified particles matches globally our expectations from a boosted thermal system. However, a detailed comparison of identified particle elliptic flow measured by the ALICE collaboration with viscous hydrodynamical calculations shows that a quantitative comparison fails for more central collisions. Including the contributions from individual hadron-hadron re-interaction cross-sections, by augmenting the viscous hydrodynamical calculations using a hadron cascade model, significantly modifies the $p_{\mathrm{T}}$-differential elliptic flow compared to pure viscous hydrodynamical calculations. However this currently does not seem to improve the description of the data. Finally, because resonances can provide more differential information on the hadronic processes, I will present in the last section the $p_{\mathrm{T}}$-differential $\phi$-meson elliptic flow.

\section{Charged Particle Elliptic Flow}

Anisotropic flow is an important observable in ultra-relativistic heavy-ion collisions as it signals the presence of multiple interactions between the constituents of the created matter. This includes possible contributions from the different phases including the hadronic phase. Therefore, anisotropic flow has been observed in nucleus-nucleus collisions from low energies up to $\sqrt{s_{\mathrm{NN}}}=2.76 \mathrm{TeV}$ at the Large Hadron Collider (LHC) [12-14]. The azimuthal anisotropic flow is usually characterized by the Fourier coefficients [15]:

$$
v_{n}=\left\langle\cos \left[n\left(\phi-\Psi_{n}\right)\right]\right\rangle,
$$

where $\phi$ is the azimuthal angle of the particle, $\Psi_{n}$ is the azimuthal angle of the plane of symmetry, and $n$ is the order of the harmonic. The second Fourier coefficient $v_{2}$ is called elliptic flow [16].
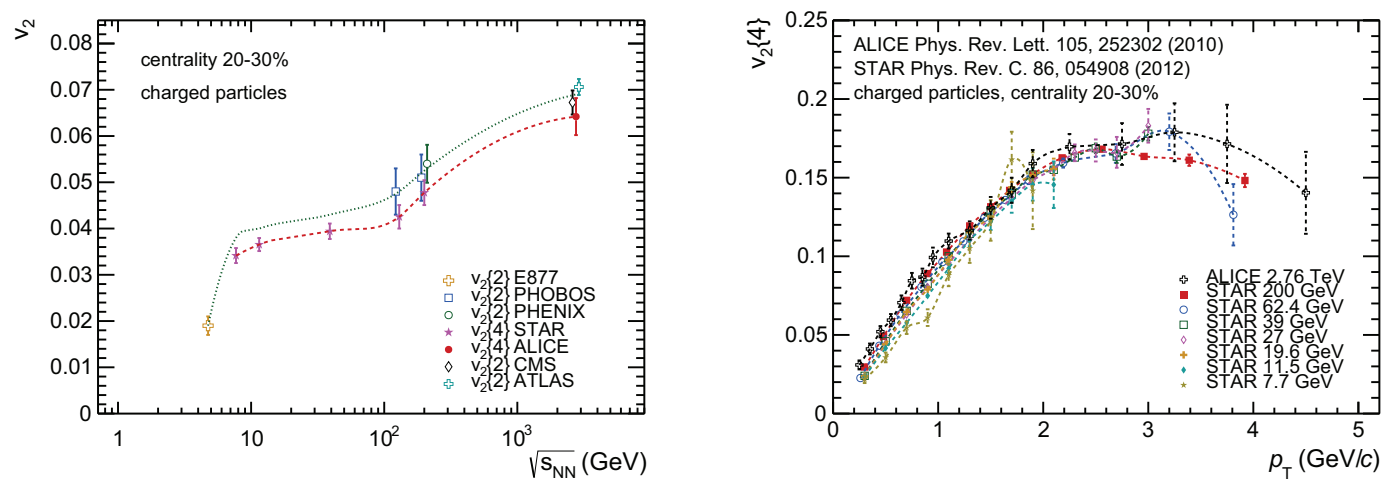

Figure 2. Left: Elliptic flow for charged particles as function of center of mass energy. Right: The charged particle $p_{\mathrm{T}}$-differential elliptic flow as function of center of mass energy $[14,17,18]$.

In the left panel of Fig. 2 the elliptic flow as function of center of mass energy is plotted for charged hadrons. Experimentally, because the planes of symmetry $\Psi_{n}$ in Eq. 1 are not known, the anisotropic flow coefficients are estimated from measured correlations between the observed particles. Here the elliptic flow estimated from two- and four-particle correlations is denoted by $v_{2}\{2\}$ and $v_{2}\{4\}$, respectively. The $v_{2}$ obtained from two-particle correlations is in general not completely due to collective 
effects, it also contains contributions from so-called non-flow (these are other sources of azimuthal correlations for instance due to jets and resonance decays). Experimentally they are suppressed as much as possible using kinematic cuts between the correlated particles. For the remaining significant difference observed between flow estimates from two- and four-particle correlations at LHC and RHIC energies, we have strong evidence that this is mainly due to event-by-event fluctuations in the elliptic flow.

The figure shows an interesting center of mass dependence of the elliptic flow which, with the recent addition of the LHC and RHIC beam energy scan results, provides tantalising evidence for a slow increase of the elliptic flow between 7.7 and $130 \mathrm{GeV}$ followed by a much steeper increase from the top RHIC to LHC energy. This behaviour is similar to what has been observed in the slopes of the particle spectra and resembles predictions by L. van Hove for the $\left\langle p_{\mathrm{T}}\right\rangle$ [19].

The right panel of Fig. 2 shows the $p_{\mathrm{T}}$-differential elliptic flow of charged particles for center of mass energies ranging from $7.7 \mathrm{GeV}$ to $2.76 \mathrm{TeV}$. Carefully examining the data, an increase of the $p_{\mathrm{T}}$-differential $v_{2}\{4\}$ is observed with increasing center of mass energy, however this increase is small. This shows that a significant fraction of the increasing elliptic flow is due to an increase in the $\left\langle p_{\mathrm{T}}\right\rangle$ [20]. A natural question to ask is if this is because a boosted thermal system is created for which the collective flow, and therefore the $\left\langle p_{\mathrm{T}}\right\rangle$, increases with center of mass energy. To answer this question we examine in the next section the $p_{\mathrm{T}}$-differential elliptic flow in much more detail with measurements of particles with very different masses.

\section{Identified Particle Elliptic Flow}

The modification to the $p_{\mathrm{T}}$-differential $v_{2}$ due to an increase in collective flow, i.e. both the radial flow, $\beta_{0}$, and its azimuthal variation, $\beta_{2}$, with increasing center of mass energy can be illustrated using a so-called blast-wave parameterisation. While the blast-wave model is simple and does not contain any dynamical information, it can be used to describe main features of the spectra, anisotropic flow and femtoscopy with a few parameters. The main model parameters are the kinetic freeze-out temperature, $T_{\text {kin }}$, the radial flow, its azimuthal variation and, the source density at freeze-out [21, 22].
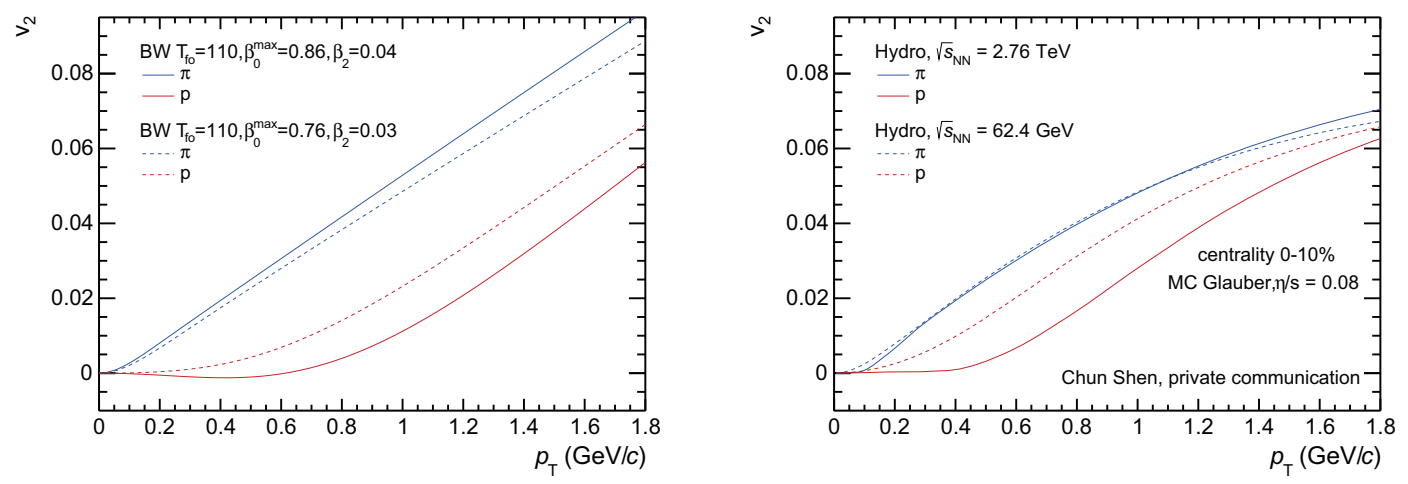

Figure 3. Left: Blast-wave results for identified particle $p_{\mathrm{T}}$-differential $v_{2}$ for two systems with different collective flow. Right: Viscous hydrodynamical calculations of identified particle $p_{\mathrm{T}}$-differential $v_{2}$ at two collision energies. 
Fig. 3 (left) shows the $p_{\mathrm{T}}$-differential $v_{2}$ from the blast-wave model for two different magnitudes of the collective flow and its azimuthal variation with all other parameters fixed. The plot clearly shows a characteristic mass splitting in the $p_{\mathrm{T}}$-differential $v_{2}$ for pions and protons. The mass splitting originates from an interplay between the blueshift generated by the radial flow and its azimuthal variation. At low- $p_{\mathrm{T}}$ we can express the $p_{\mathrm{T}} \approx p_{\mathrm{T}}^{\text {th }}+m c \beta$ as the sum of a thermal contribution (independent of the mass of the hadron) and a radial flow component with flow velocity $\beta$ (this part is proportional to the mass of the hadron). The blueshift generates a mass dependent modification to the hadron $p_{\mathrm{T}}$-spectra and determines the magnitude of the mass splitting in the $p_{\mathrm{T}}$-differential $v_{2}$ of the hadrons. The blast-wave curves indeed show that the mass splitting increases from the dashed curves (smaller radial flow) to the solid curves (larger radial flow).

In relativistic viscous hydrodynamics, a dynamical model of the system evolution, the $p_{\mathrm{T}^{-}}$ differential $v_{2}$ depends on many more parameters, e.g. initial conditions, equation of state, transport parameters such as $\eta / s$ and freeze-out conditions. At different center of mass energies, some of these parameters are typically constrained by the measured integrated charged hadron yields and pion spectra in central collisions. As a result the centrality and mass dependence of the $p_{\mathrm{T}}$-differential $v_{2}$ can be used to check the validity of the model and to constrain the main transport parameters. More recently, measurements of the event-by-event fluctuations in $v_{2}$ and additional higher harmonics, such as $v_{3}$, are used to provide stringent constraints on the initial conditions.

In the right panel of fig. 3 the $p_{\mathrm{T}}$-differential $v_{2}$ for pions and protons is plotted from viscous hydrodynamical calculations, using MC Glauber initial conditions and a kinetic viscosity $\eta / s=0.08$. These detailed model calculations also clearly show the characteristic mass splitting, which increases with increasing center of mass collision energy (dashed and solid curves are examples for RHIC and LHC energies, respectively).
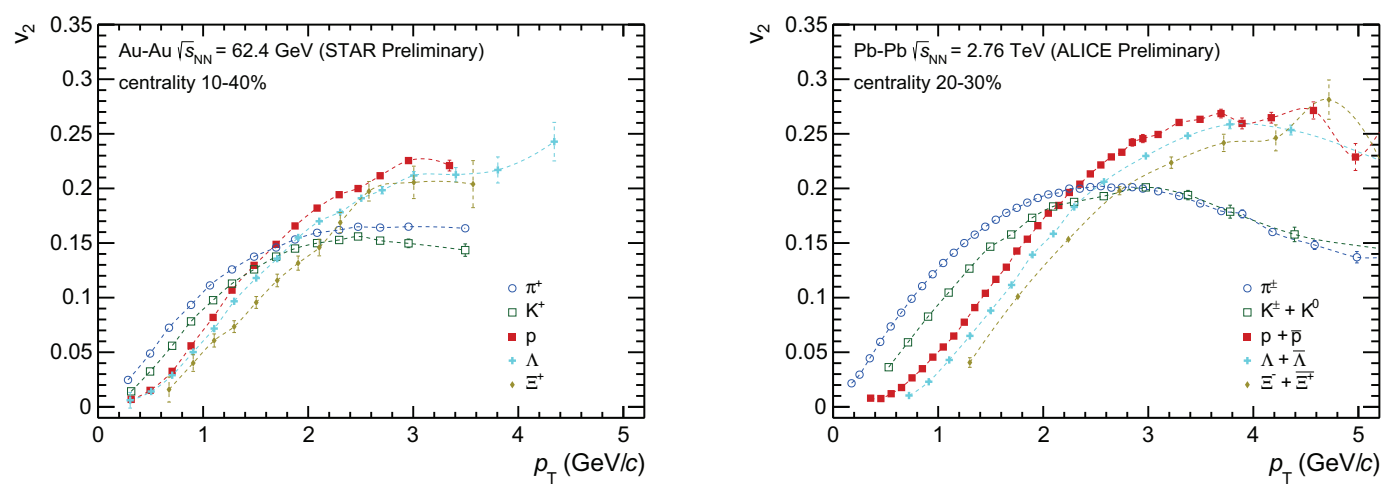

Figure 4. Left: STAR $p_{\mathrm{T}}$-differential identified particle $v_{2}$ for Au-Au collisions at $\sqrt{s_{\mathrm{NN}}}=62.4 \mathrm{GeV}$. Right: ALICE $p_{\mathrm{T}}$-differential identified particle $v_{2}$ for $\mathrm{Pb}-\mathrm{Pb}$ collisions at $\sqrt{s_{\mathrm{NN}}}=2.76 \mathrm{TeV}$ [23]

Recently the STAR collaboration has performed anisotropic flow measurements in a range of center of mass energies as part of their beam energy scan program. In Fig. 4 (left) the $p_{\mathrm{T}}$-differential $v_{2}$ is shown for pions, kaons, protons, lambda and cascade, measured at $\sqrt{s_{\mathrm{NN}}}=62.4 \mathrm{GeV}$. For $p_{\mathrm{T}}<1.5 \mathrm{GeV} / c$ a clear mass ordering is observed for the particles which vary in mass by an order of magnitude. In the right panel of Fig. 4 the measurements at $\sqrt{s_{\mathrm{NN}}}=2.76 \mathrm{TeV}$, by the ALICE 
collaboration, are plotted. The same mass ordering, for $p_{\mathrm{T}}<2 \mathrm{GeV} / c$, is again observed, however we see that the mass splitting is much more pronounced at a larger center of mass energy.

Ideal hydrodynamics predicts that the mass splitting pattern persists even at large values of $p_{\mathrm{T}}$ in contrast to what is observed in the data at RHIC and the LHC, this is apparent from Fig. 4 above $p_{\mathrm{T}} \approx 2 \mathrm{GeV} / c$. An elegant explanation of the particle type dependence and magnitude of $v_{2}$ at larger $p_{\mathrm{T}}$ is provided by the coalescence picture [25].
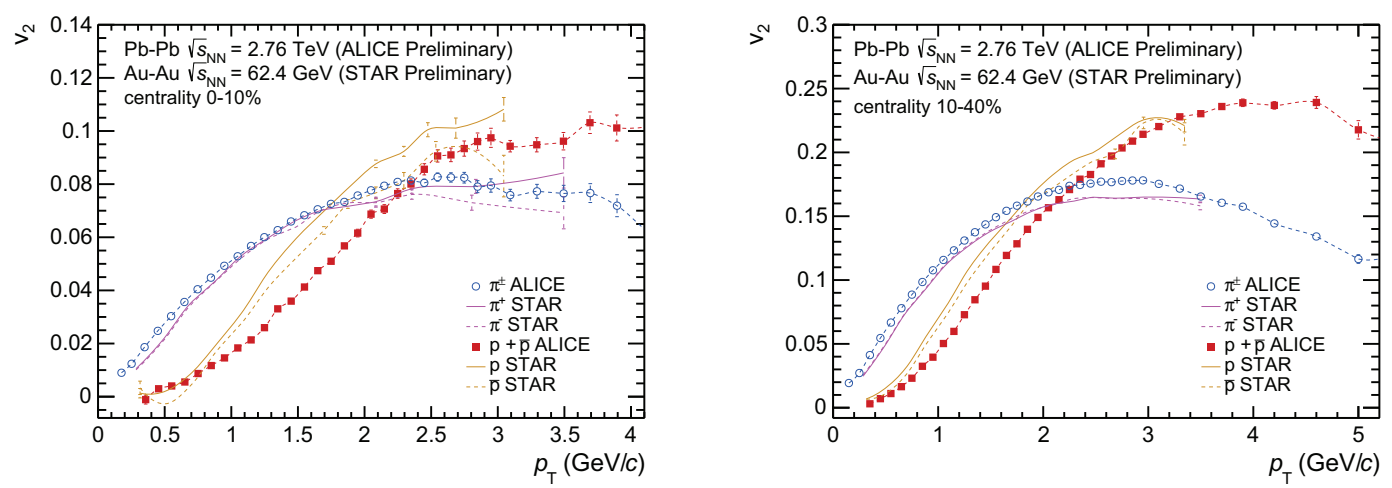

Figure 5. Comparison of $p_{\mathrm{T}}$-differential $v_{2}$ at $\sqrt{s_{\mathrm{NN}}}=62.4 \mathrm{GeV}$ [24] and $2.76 \mathrm{TeV}$ [23] for different particle species. Left: for the central 0-10\% collisions. Right: for the collisions in the 10-40\% centrality percentile.

To compare the collision energy dependence of the mass splitting we compare for two centralities the $p_{\mathrm{T}}$-differential $v_{2}$ for pions and protons measured by STAR and ALICE. Figure 5 (left) shows the comparison for the $0-10 \%$ centrality percentile. Because at RHIC energies we observe a small difference between the particle and antiparticle anisotropic flow [26], the STAR results are plotted separately for $\pi^{+}, \pi^{-}$and $p, \bar{p}$. In the right panel of Fig. 5 the same comparison is shown for more peripheral, 10-40\%, collisions. This comparison clearly illustrates the significant increase of the mass splitting between pions and protons as function of collision energy, for both centrality ranges. Such an increase is expected if the collective flow increases with collision energy and is therefore in qualitative agreement with hydrodynamical model calculations.

First preliminary measurements of the $p_{\mathrm{T}}$-differential $v_{2}$ at $\sqrt{s_{\mathrm{NN}}}=2.76 \mathrm{TeV}$ for pions, kaons and protons were presented and compared to viscous hydrodynamical model predictions at QM2011 [27]. For mid-central collisions, 40-50\%, a fair agreement between the model calculations and the data was observed. In Fig. 6 the comparison between viscous hydrodynamical calculations and the data is shown, here also including the lambdas and cascades. Also for the lambdas and cascades there is a fair agreement between data and theory.

However, for more central collisions a clear discrepancy between data and theory was observed for the protons. This discrepancy, also observed in the yields and spectra of the protons and antiprotons, is part of what is called the proton puzzle at the LHC. Recent ALICE measurements of the $p_{\mathrm{T}}$-differential $v_{2}$, which now include more particles, show that this discrepancy is not only observed for the protons but also for other baryons.

Although there is consensus that much of the dynamical evolution can be modelled by relativistic viscous hydrodynamics, the clear deviations between data and the model, such as for the $p_{\mathrm{T}^{-}}$ differential $v_{2}$ of baryons in more central collisions, show that not all relevant ingredients are in place. 

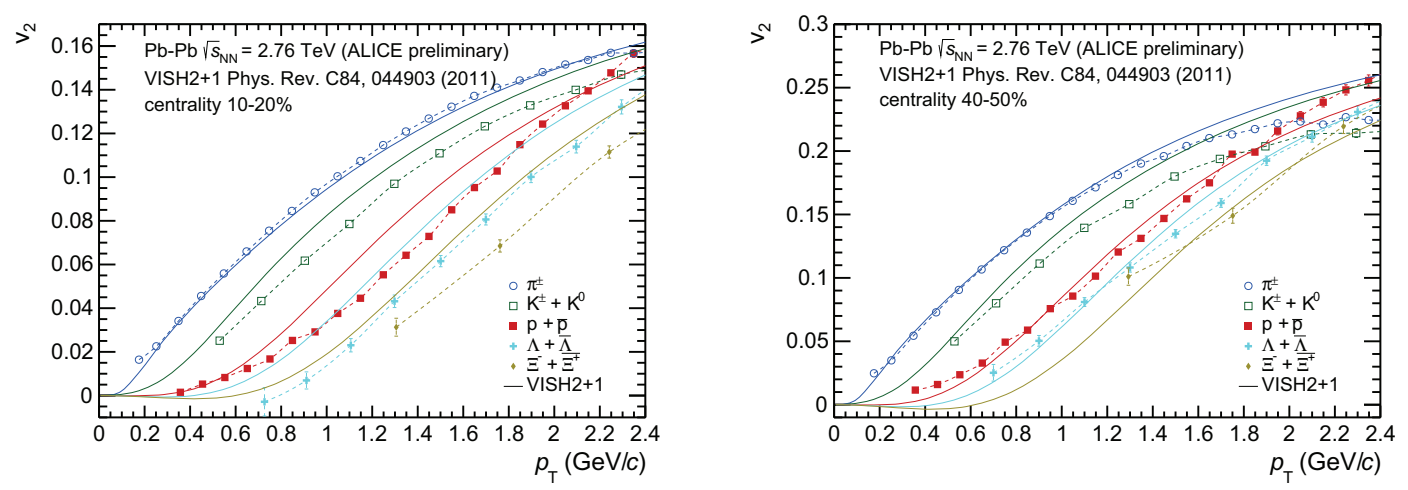

Figure 6. Comparison between ALICE measurements of $p_{\mathrm{T}}$-differential $v_{2}$ at $\sqrt{s_{\mathrm{NN}}}=2.76 \mathrm{TeV}$ [23] and viscous hydrodynamical model predictions. Left: for the central 10-20\% collisions. Right: for the collisions in the range of $40-50 \%$ centrality percentile. The dashed lines connect the datapoints.
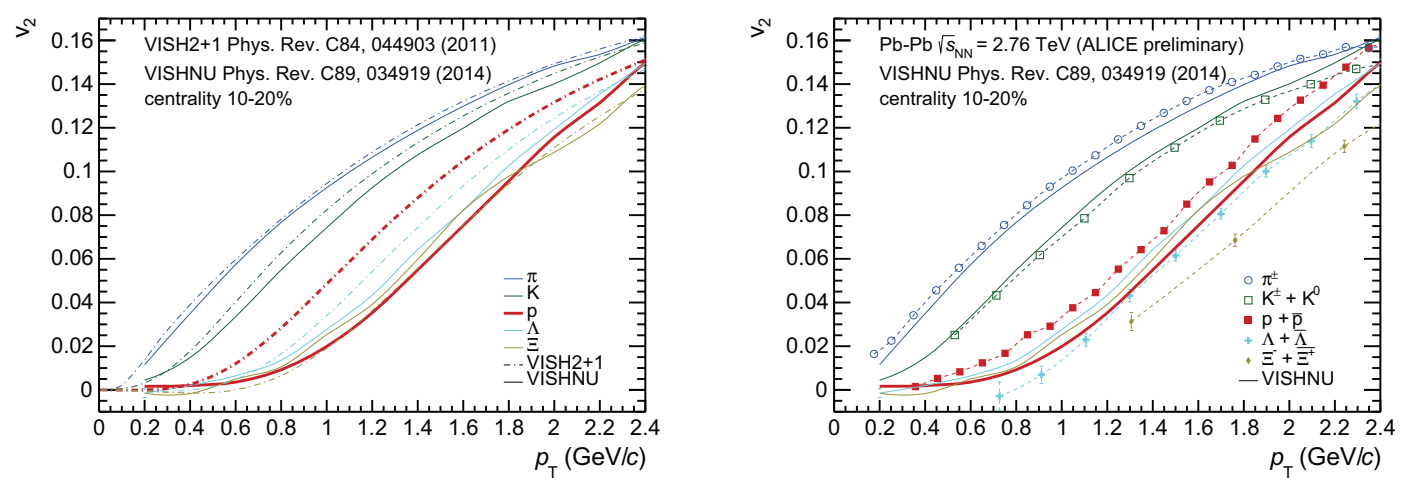

Figure 7. Left: Viscous hydrodynamical calculations (VISH2+1) compared to viscous hydrodynamical calculations augmented with a hadronic cascade afterburner (VISHNU). Right: VISHNU model calculations compared to ALICE measurements [23] of $p_{\mathrm{T}}$-differential $v_{2}$ at $\sqrt{s_{\mathrm{NN}}}=2.76 \mathrm{TeV}$ for central $10-20 \%$ collisions.

Augmenting the viscous hydrodynamical model calculations with a hadron cascade afterburner is one of the most obvious possibilities to improve our understanding and description of the data.

In the left panel of Fig. 7 viscous hydrodynamical calculations with and without a hadronic cascade afterburner, VISHNU and VISH2+1 respectively, are compared. The increase in mass splitting between the pions and kaons for VISHNU (solid curves) compared to VISH2+1 (dashed curves) illustrates the larger radial flow in the VISHNU calculations due to the contribution of the hadronic cascade. Between the pions and lambdas a similar increase is observed. However it is seen that the mass splitting between the pions and cascades does not change much. This can be understood because in most hadronic cascade models a small hadronic re-interaction cross section is assigned to the cascade baryon. In a hadron cascade model such as URQMD the magnitude of the hadronic re- 
interaction cross section is, if these cross sections are not measured, related to the number of strange quarks contained in the hadron (additive quark model). In that case, the amount of radial flow that the hadrons pick up in the hadronic phase depends on their strange quark content. For the protons the most dramatic change is observed, as they are strongly coupled to the flowing matter because of their large interaction cross section with the pions. As a result of the different individual hadron-hadron reinteraction cross-sections in the hadronic phase the characteristic mass ordering, which was observed in VISH2+1, is not preserved anymore after including the hadronic cascade in VISHNU.

The comparison between the $p_{\mathrm{T}}$-differential $v_{2}$ measured by ALICE and the VISHNU model is shown in the right-panel of Fig. 7. While VISHNU gives a better description of the kaons, and partially also for the lambdas, it does not improve the description of the cascades. For the protons the modification of the $p_{\mathrm{T}}$-differential $v_{2}$ is so large that, compared to VISH2+1, it overcompensates for the original difference and again fails to describe the data. In particular the breaking of the mass ordering as seen in VISHNU is not observed in the data.

Clearly, the current implementations of viscous hydrodynamics coupled to a hadron cascade do not describe better the $p_{\mathrm{T}}$-differential $v_{2}$ of the different particle species in more central collisions. Therefore, it is currently still an open question what the origin is of the observed larger radial flow in more central collisions. Possible contributions could come from pre-equilibrium flow (e.g. AdS/CFT [28, 29]), contributions from a different baryon production mechanism [30, 31], or perhaps just from improving our knowledge of some of the hadronic re-interaction cross sections.

\section{$4 \phi$-Meson Elliptic Flow}

Among the various hadrons measured in heavy-ion collisions, resonances can provide more differential information on the hadronic processes because of their different lifetimes and re-interaction cross sections. An example is the observed non-constant $K^{* 0} / K^{-}$ratio as function of centrality [3]. This deviates from thermal and statistical model predictions and could be explained by contributions from hadronic re-scattering and regeneration.

Because the amount of hadronic re-scattering and the amount of radial flow picked up in the hadronic phase is assumed to depend on the strange quark content the $\phi$-meson spectra and anisotropic flow are expected to be sensitive measurements $[32,33]$. The $\phi$-meson contains a strange and antistrange quark and therefore its $p_{\mathrm{T}}$-differential $v_{2}$ is expected to be primarily generated in the partonic phase. The $\phi$-meson $p_{\mathrm{T}}$-differential $v_{2}$ is expected, in case of mass ordering, to be in-between the protons and lambdas. However if the contribution to the collective flow depends strongly on the different individual hadron-hadron re-interaction cross-sections the mass ordering will be broken because the lambdas and in particular the protons will pick up a significant additional amount of flow.

Figure 8 shows the $\phi$-meson $p_{\mathrm{T}}$-differential $v_{2}$ for more central, 10-20\%, and more peripheral, $40-50 \%$, collisions. Below $p_{\mathrm{T}}=2.5 \mathrm{GeV} / c$ the $\phi$-meson follows within the relatively large uncertainties the mass hierarchy for both centralities. However, for the lowest $p_{\mathrm{T}}$ bin there is an indication that the $\phi$-meson $v_{2}$ is larger than the proton $v_{2}$. Unfortunately, the uncertainties in the ALICE $\phi$ meson $v_{2}$ measurements are currently still too large at low- $p_{\mathrm{T}}$ to constrain better the possible hadronic contribution.

\section{Summary}

In these proceedings I have shown that, with the recent measurements from the LHC and from the RHIC beam energy scan, we observe tantalising evidence for a change in slope of the energy dependence of the integrated elliptic flow. From the $p_{\mathrm{T}}$-differential $v_{2}$ measurements of different particle 

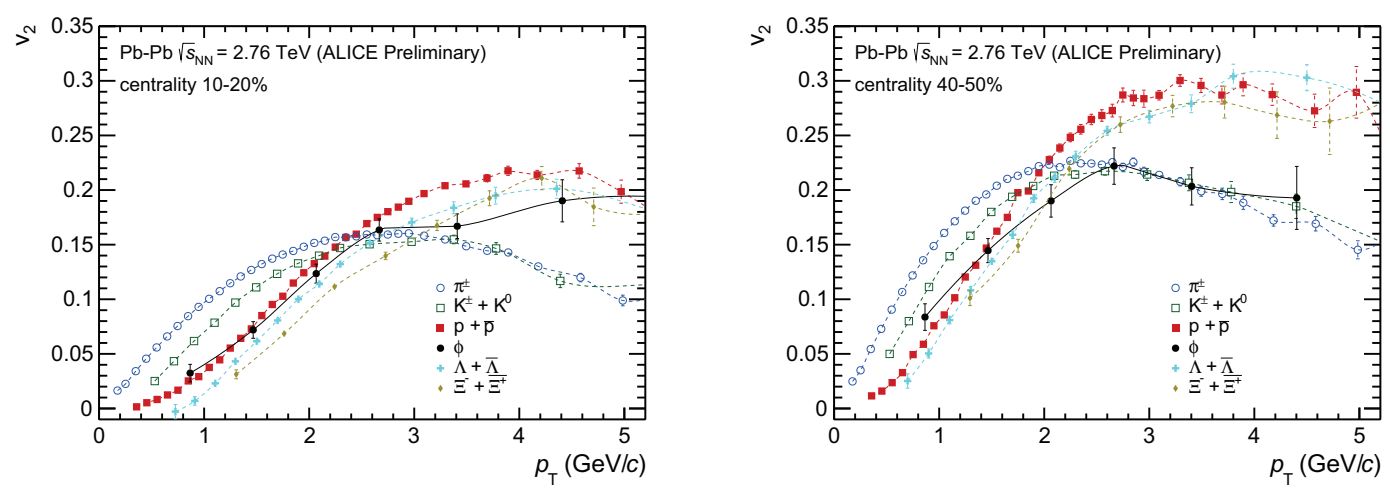

Figure 8. The $\phi$-meson $p_{\mathrm{T}}$-differential $v_{2}$ compared to other identified particles for two collision centralities (from [23]).

species we see that this can be understood due to increasing collective flow with increasing center of mass collision energy. A comparison between the measurements and viscous hydrodynamical model calculations with and without a hadronic afterburner shows that currently the contributions from the hadronic phase are not understood yet.

\section{Acknowledgements}

I would like to thank the organizers of the conference, in particular Angela Badala for insisting that I would prepare this talk and also for the beautiful birthday cake. For the contributions to the figures and for the stimulating discussions I am grateful to: Joerg Aichelin, Anton Andronic, Tomasz Bold, Panos Christakoglou, Pasi Huovinen, Wojciech Florkowski, Ulrich Heinz, Hannah Petersen, Jurgen Schukraft, Alexander Schmah, Chun Shen, Shusu Shi, Huichao Song, Jan Steinheimer, Sergei Voloshin, Klaus Werner, $\mathrm{Nu} \mathrm{Xu}$, and You Zhou.

\section{References}

[1] http://u.osu.edu/vishnu/2014/08/06/sketch-of-relativistic-heavy-ion-collisions/

[2] J. Stachel, A. Andronic, P. Braun-Munzinger and K. Redlich, J. Phys. Conf. Ser. 509, 012019 (2014) [arXiv:1311.4662 [nucl-th]].

[3] B. Abelev et al. [ALICE Collaboration], Phys. Rev. C 91, 024609 (2015) [arXiv:1404.0495 [nucl-ex]].

[4] B. Abelev et al. [ALICE Collaboration], Phys. Rev. C 88, no. 4, 044910 (2013) [arXiv:1303.0737 [hep-ex]].

[5] F. Becattini, M. Bleicher, T. Kollegger, M. Mitrovski, T. Schuster and R. Stock, Phys. Rev. C 85, 044921 (2012) [arXiv:1201.6349 [nucl-th]].

[6] V. Begun, W. Florkowski and M. Rybczynski, Phys. Rev. C 90, no. 5, 054912 (2014) [arXiv:1405.7252 [hep-ph]].

[7] F. Becattini, M. Bleicher, T. Kollegger, T. Schuster, J. Steinheimer and R. Stock, Phys. Rev. Lett. 111, 082302 (2013) [arXiv:1212.2431 [nucl-th]]. 
[8] H. Song and U. W. Heinz, Phys. Lett. B 658, 279 (2008) [arXiv:0709.0742 [nucl-th]]. H. Song and U. W. Heinz, Phys. Rev. C 77, 064901 (2008) [arXiv:0712.3715 [nucl-th]].

[9] H. Song and U. W. Heinz, Phys. Rev. C 78, 024902 (2008) [arXiv:0805.1756 [nucl-th]].

[10] H. Song, S. A. Bass and U. Heinz, Phys. Rev. C 83, 024912 (2011) [arXiv:1012.0555 [nucl-th]].

[11] S. A. Bass, M. Belkacem, M. Bleicher, M. Brandstetter, L. Bravina, C. Ernst, L. Gerland and M. Hofmann et al., Prog. Part. Nucl. Phys. 41, 255 (1998) [Prog. Part. Nucl. Phys. 41, 225 (1998)] [nucl-th/9803035].

[12] S. A. Voloshin, A. M. Poskanzer and R. Snellings, in Landolt-Boernstein, Relativistic Heavy Ion Physics, Vol. 1/23, p 5-54 (Springer-Verlag, 2010)

[13] U. Heinz and R. Snellings, Ann. Rev. Nucl. Part. Sci. 63, 123 (2013) [arXiv:1301.2826 [nuclth]].

[14] K. Aamodt et al. [ALICE Collaboration], Phys. Rev. Lett. 105, 252302 (2010)

[15] S. Voloshin and Y. Zhang, Z. Phys. C 70665 (1996)

[16] J. Y. Ollitrault, Phys. Rev. D 46, 229 (1992)

[17] S. Shi [STAR Collaboration], Nucl. Phys. A 904-905, 895c (2013) [arXiv:1210.4607 [nucl-ex]].

[18] L. Adamczyk et al. [STAR Collaboration], Phys. Rev. C 86, 054908 (2012) [arXiv:1206.5528 [nucl-ex]].

[19] L. van Hoven, Phys. Lett. B 118, 138 (1982)

[20] B. B. Abelev et al. [ALICE Collaboration], Phys. Lett. B 727, 371 (2013) [arXiv:1307.1094 [nucl-ex]].

[21] C. Adler et al. [STAR Collaboration], Phys. Rev. Lett. 87, 182301 (2001) [nucl-ex/0107003].

[22] F. Retiere and M. A. Lisa, Phys. Rev. C 70, 044907 (2004) [nucl-th/0312024].

[23] B. B. Abelev et al. [ALICE Collaboration], arXiv:1405.4632 [nucl-ex].

[24] L. Adamczyk et al. [STAR Collaboration], Phys. Rev. C 88, 014902 (2013) [arXiv:1301.2348].

[25] D. Molnar and S. A. Voloshin, Phys. Rev. Lett. 91, 092301 (2003) [nucl-th/0302014].

[26] L. Adamczyk et al. [STAR Collaboration], Phys. Rev. Lett. 110, 142301 (2013) [arXiv:1301.2347 [nucl-ex]].

[27] M. Krzewicki [ALICE Collaboration], J. Phys. G 38, 124047 (2011) [arXiv:1107.0080 [nuclex]].

[28] W. van der Schee, P. Romatschke and S. Pratt, Phys. Rev. Lett. 111, no. 22, 222302 (2013) [arXiv:1307.2539].

[29] M. Habich, J. L. Nagle and P. Romatschke, arXiv:1409.0040 [nucl-th].

[30] K. Werner, I. Karpenko, M. Bleicher, T. Pierog and S. Porteboeuf-Houssais, Phys. Rev. C 85, 064907 (2012) [arXiv:1203.5704 [nucl-th]].

[31] Klaus Werner, these proceedings.

[32] T. Hirano, U. W. Heinz, D. Kharzeev, R. Lacey and Y. Nara, Phys. Rev. C 77, 044909 (2008) [arXiv:0710.5795 [nucl-th]].

[33] H. Song, S. Bass and U. W. Heinz, Phys. Rev. C 89, no. 3, 034919 (2014) [arXiv:1311.0157 [nucl-th]]. 